\title{
Diabetic Retinopathy - Feature Extraction and Classification using Adaptive Super Pixel Algorithm
}

\author{
Balambigai Subramanian, V. Saravanan, Rudra Kalyan Nayak, T. Gunasekaran, S. Hariprasath
}

\begin{abstract}
Diabetic Retinopathy is an ocular manifestation of diabetes. The longer a person has diabetes, higher are the chances of having diabetic retinopathy in their visual system. Hence the objective of this research work is to propose an automated, suitable and sophisticated approach using image processing so that diabetic retinopathy can be detected at early levels easily and damage to retina can be minimized. A vital point of diabetic retinopathy that it causes detectable changes in the blood vessels of the retina. The focal blurred edges are detected so as to dismiss the false alarms. A two-level approach is used here to classify data. Firstly, optimal features are extracted from the training data and secondly, the classification is done by the use of the adaptive super pixel algorithm and then the test data is analyzed. Adaptive super pixel algorithm can adjust the weights of various features based on their discriminating ability. After the application of algorithm, the diabetic eye is detected by means of various parameters like colour, texture, spatial distance, contour, mean, standard deviation, entropy and maximum pixel points. This research can aid the doctor for easy detection of the disease as it given an accuracy of about $\mathbf{9 8 . 3 3 \%}$.
\end{abstract}

Keywords: Retinopathy, fundus, adaptive super pixel, classification

\section{INTRODUCTION}

$\mathrm{D}$ iabetes occurs among various people due to various factors like sedentary life style, stress and eating habits. Diabetic Retinopathy usually happens if a person has diabetes for more than ten years or more. Diabetic Retinopathy (DR), causes around five percent of blindness among people and this is one of the major reason that causes blindness among diabetics. According to World health Organization (WHO) estimation, 425 million of world population is having the diabetes [1] .Research indicates that

Revised Manuscript Received on December 30, 2019.

* Correspondence Author

Dr. Balambigai Subramanian, Associate Professor, Department of Electronics and Communication Engineering, Kongu Engineering College. E-mail: sbalambigai@gmail.com

Dr. V. Saravanan, Associate Professor, Department of Computer Science and Engineering, K L Deemed to be University, Guntur (Andhra Pradesh) India.

Dr. Rudra Kalyan Nayak, Associate Professor, Department of Computer Science and Engineering, K L Deemed to be University, Vaddeswaram (Andhra Pradesh) India.

Dr. T. Gunasekaran, Department of Electronics and Communication Engineering, Higher College of Technology, Muscat

Mr. S. Hariprasath ,Assistant Professor in Department of Electronics and Communication Engineering, Saranathan College of Engineering, Trichy (Tamil Nadu) India.

(C) The Authors. Published by Blue Eyes Intelligence Engineering and Sciences Publication (BEIESP). This is an open access article under the CC BY-NC-ND license (http://creativecommons.org/licenses/by-nc-nd/4.0/) progress of the reduction of can be reduced or eradicated if diabetic retinopathy is found at an early stage. It is found that large number of people having diabetic retinopathy are being tested for this disease by manual method by trained opthamalogists which is a time consuming process and sometimes delay in diagnosis may lead to delay in treatment to patients. Diabetes is usually caused when insulin is produced less in body or if the human body develops resistance to glucose or when both the causes occur simulataneously[2].A person with diabetes has a condition in which the quantity of glucose in the blood is too elevated (hyperglycemia). This is because the body does not produce enough insulin, produces no insulin, or has cells that do not respond properly to the insulin the pancreas produces[3]. Research says for about $90 \%$ of diabetics, eye disease can be managed through better medical treatment and periodic checkup of the eyes. Image processing is used by the ophthalmologists to diagnose the various eye diseases. In India, it is predicted that 79.4 million people may suffer from diabetes, out of which about 75 percent of people those who have this disease for more than 20 years may have certain form of problem in vision due to diabetic retinopathy[4] . The blood vessel changes in the retina of the eye gives information about change in the values of some of the features of the eye, such as blood vessels of the eyes , optic disc (OD), that are helpful to find the occurrence of diabetic retinopathy (DR) or any other eye diseases. Poor people are easily affected by diabetic retinopathy due to the non availability of screening, early diagnosis and treatment. Many screening tools are available to diagnose diabetic retinopathy. Fundus cameras capture digital image of the blood vessels in the retina and sometimes they may be having excessibe brightness etc that reduce the quality of the image. Hence, there is a need to enhance the images by the use of image enhancement. Features of the eye that help to detect the retinopathy are blood vessels, textures microaneurysms, exudes etc. These features increase in size and may destruct the capillaries causing blood leakage in the eye leading to blindness.. Hence, the objective of this research is to detect diabetic retinopathy earlier to minimize the damage to retina[5].

\section{LITERATURE REVIEW}

Ronakorn Panjaphongse (2017) used the morphological operators along with support vector machine (SVM) to screen diabetic retinopathy earlier among the diabetics. Initially, pre processing of the image is done to improve image quality,followed by filtering after edge detection and morphological operations.

\section{Published By:}


The extracted features are given as input to the support vector machine classifier which differentiates between healthy and diabetic retinopathy affected eye images. The proposed method iwas verified on three datasets and this method gave an accuracy of $90 \%$. The grading of severity was based on the values of the various above mentioned parameters for the detected lesions [6].

Manoj Kakarla (2016) discussed about extracting the various features of the eye in an optimal manner, followed by feature extraction and classification of diabetes using naïve bayes classifier. This work discusses an expert system to identify the stage of diabetes based on patients test result. Before performing classification process, optimal features are extracted based on data sets for the purpose of reducing time complexity over the classification process[7]. Then the classification process is performed by naïve bayes theorem to identify each patient's stage in the progress of the disease diabetes. By implementing these concepts, the efficiency of classification increases and also reduces the time complexity for performing the classification process.

K. R. Ananthapadmanaban etal (2014) proposed the chance of occurrence of diabetic retinopathy based on classification using data mining methods[8]. In this work, diabetic retinopathy has been diagnosed earlier. The preprocessing operation improves quality of the image by reducing the effects of excessive brightness. In the second step, the optic disc has to be located and extracted from the retinal image. The third step consists of the segmentation to find the exudates . In the last step, the neural network is used for feature extraction. The final results were compared with the opinion gathered from an eye doctor and it was found that the specificity was $95 \%$ and sensitivity was $96.65 \%$.

Hilary W. Thompson (2016) proposed a novel method based on hierarchical decomposition and post filtering of edges to detect blood vessels to detect the occurrence of diabetic retinopathy at an early stage. This algorithm reduced the false decisions and was quite faster than conventional methods and needed less storage requirements[9].Ms. Bhaminee R Shetty (2017) proposed the automatic segmentation of microaneurysm using attribute filtering to reduce noise and thereby detected the exudates.Later, the feature of exudates was extracted from green channel of the RGB retinal image which was later given as input to the classifier. Statistical parameters such as mean, standard deviation, kurtosis etc were considered for classification and SVM gave sensitivity of $96.9 \%$.[10]

\section{EXISTING SYSTEM}

\subsection{NAÏVE BAYESIAN CLASSIFIER}

This method extracts the optimal features initially from the training data [19]. Later, the values of positive and negative probability is found .Later, a new dataset of same size is created to which the values of previous data set are forwarded to help in the classification process. After completion of optimal feature extraction process, that dataset is taken and sent to classification. By applying classification algorithm, each patient's stage over the diabetes can be identified. The probability formula for naïve bayes algorithm is shown in equation 3.1

$$
P(T / E)=\frac{P(E / T) \times P(T)}{P(E / T) \times P(T)+P(E /-T \times P(-T))}
$$

\subsection{SUPPORT VECTOR MACHINE}

Support Vector Machine used points in space to represent the features so that the data can be mapped into different categories with clear boundary gap between them [14]. SVM is more suitable to analyze the fundus retinal image and to classify them into various classes such as normal or non-proliferative diabetic retinopathy or proliferative diabetic retinopathy. Using SVM classifier that uses a non linear kernel function, the accuracy level increases due to the techniques used for extraction and classification.

\subsection{K-NN CLASSIFIER}

K-Nearest Neighbour (KNN) algorithm classifies an object to an appropriate class based on the $\mathrm{K}$ value. Generally, $\mathrm{K}$ value is considered to be one for classification of retinal image. The graphs indicate the "variation" between the statistical parameters, which is the base for a K-NN classifier system to find out nearest neighbour for a new image [20]. This variation is found better for the statistical parameters extracted by using K-means classifier and this is best suited for classifying a retinal image as healthy or abnormal.

\section{PROPOSED SYSTEM}

\subsection{PROPOSED METHODOLOGY}

In this proposed work, the input images of size 256x256 pixels are pre-processed to convert the input colour images in RGB to gray images because inherent complexity of gray image is less than the colour images. Also, gray scale reduces the complexity to one dimension from three dimension. RGB segmentation is used for dividing image into several region with their own characteristics for extracting useful target like lines and curves of an image. Image enhancement allows to brighten an image and makes it easier to identify the key features in preprocessing. The block diagram which is used for this research is shown in figure 4.1.

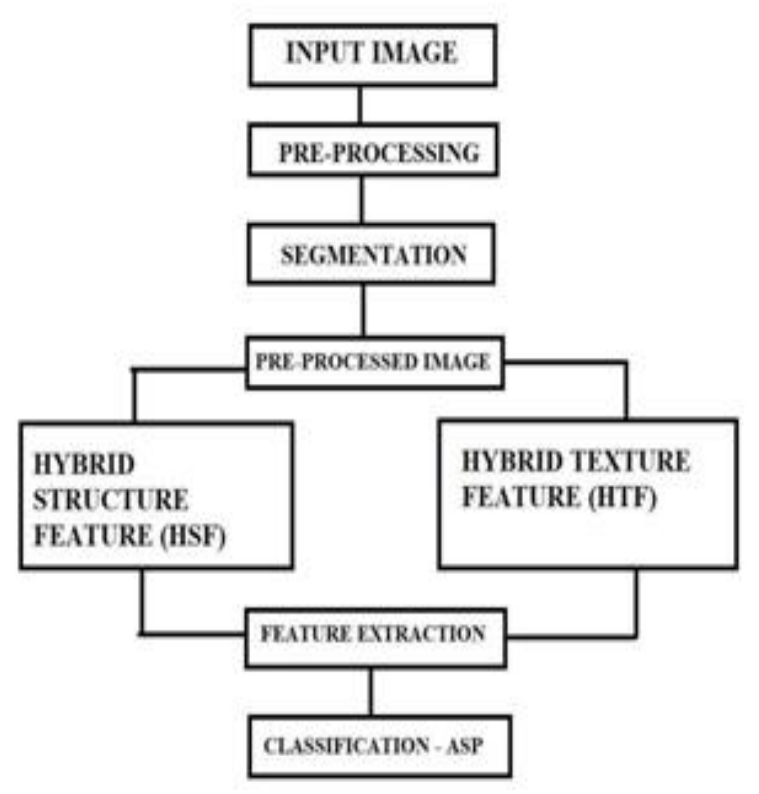

Figure 4.1 Block diagram of proposed system 
Segmentation of preprocessed images is done by using DWT (Discrete Wavelet Transform) using Haar family wavelets. The preprocessed image is classified into two types based on their nature by Hybrid Structure Feature (HSF) and Hybrid Texture Feature (HTF). The HSF performs optical disc removal, threshold point calculation. And to find the region of interest. HTF involves in blood vessel removal and dots part detection.In feature extraction the black points, white points, mean error, standard deviation, entropy and maximum pixel points are extracted.

\subsection{ADAPTIVE SUPER PIXEL ALGORITHM}

Super pixel segmentation uses super pixels that show clear entity for human eye.This algorithm leads to cost reduction as super pixels are used and processing of super pixels become easier. Boundary are clearly marked based on regularity of the super pixels .Design of adaptive super pixel algorithm is due to the following factors such as colour difference is not sufficient to produce meaningful entity in low contrast images and various colours have various values for its parameters of texture, colour, contour etc. The flow chart of the above algorithm is shown in figure 4.2.

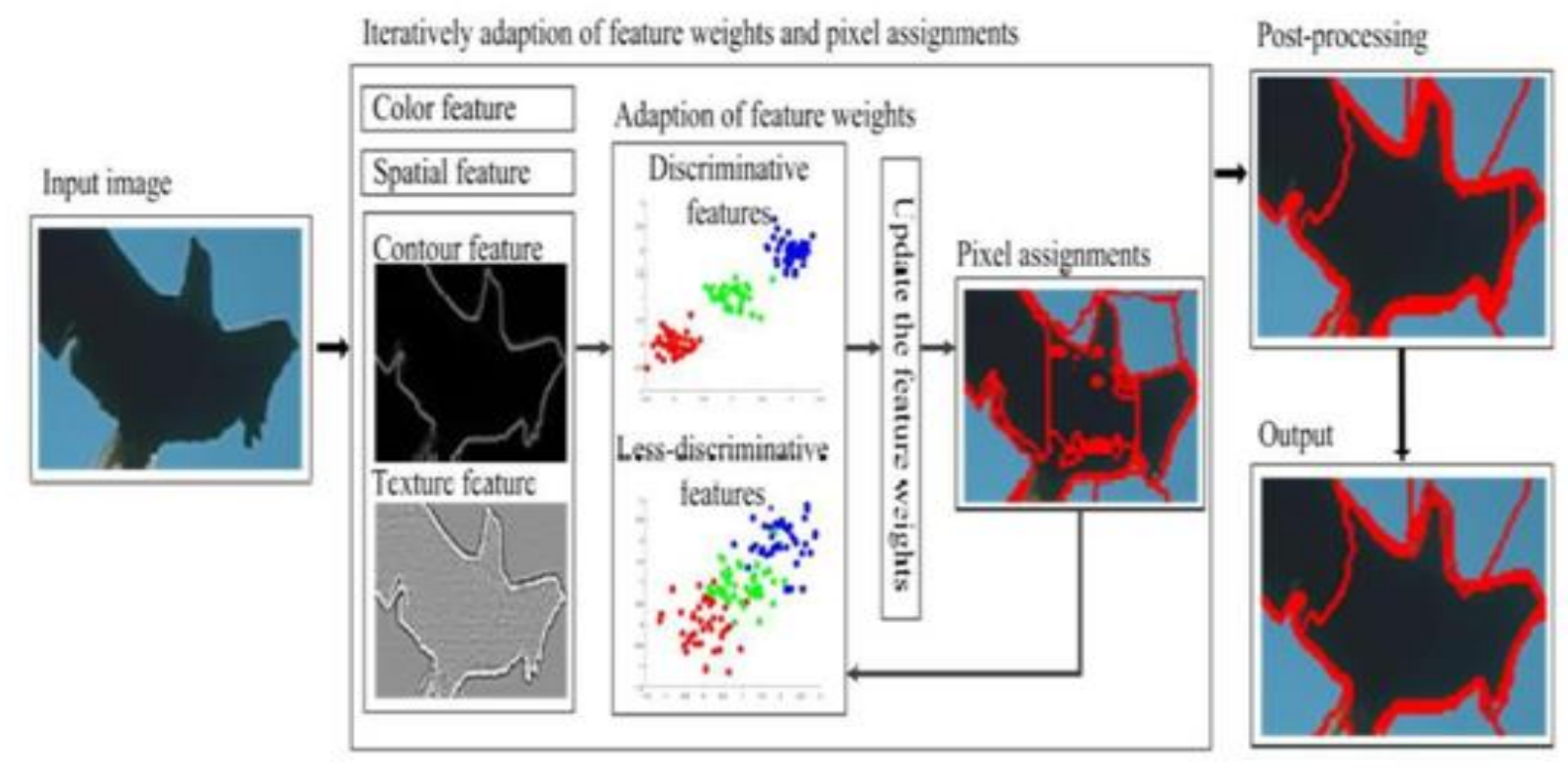

Figure 4.2 Flow chart of adaptive super pixel algorithm

The novelties of adaptive super pixel lie in:

- Consistency of Perception

- Adaptability of content

- Simplicity and efficiency

\subsection{THE FEATURES AND DISTANCE MEASURE}

Colour difference $\mathbf{d}_{\mathbf{c}}$ : It is calculated using equation 4.1 as referred in [23]

$d_{c}=\sqrt{\left(\frac{\Delta l}{k_{l} s_{l}}\right)^{2}+\left(\frac{\Delta c}{k_{c} s_{c}}\right)^{2}+\left(\frac{\Delta h}{k_{h} s_{h}}\right)^{2}}$,

where $\Delta l, \Delta_{c}$, and $\Delta h$ represents the differences of l, c, and $h$ between two pixels

- Significance: Colour difference is used to find the contrast of each pixels.

\section{Spatial distance:}

- Significance: Spatial distance is used to find the length of a straight line in a 3-D space i.e. the distance between the two-pixel points is calculated.

\section{Image gradient:}

- Significance: Image gradient is useful to find the changes in directional intensity of an retinal image .

Weber local descriptor:

- Significance: Weber local descriptor finds patterns and difference in texture to find the features.

Distance D between pixels is shown in equation 4.2.

$D=\sqrt{\omega_{c}\left(d_{c}\right)^{2}+\omega_{s}\left(d_{s}\right)^{2}+\omega_{g}\left(d_{g}\right)^{2}+\omega_{u}\left(d_{u}\right)^{2}}, \ldots$

\subsection{IMAGE SEGMENTATION}

Image segmentation divides the image into many segments to simplify or change its representation so that its analysis becomes easier. Labels are assigned so that same label contains the pixels with similar characteristics. The steps involved are

i) Converting the RGB image to grayscale to retain luminance and remove hue and saturation.

ii) Improving the contrast by the method of histogram equalization.

iii)Next, image enhancement is done by contrast adjustment, morphological filtering so as to Return a modified version of the original retinal image.

iv) Optic disk present on the retina is very bright and it has to be extracted using feature extraction methods to identify the blood vessels.

v) Blood vessels are extracted to be examined for identifying various eye diseases such as diabetic retinopathy.

vi) The next step is the classification of exudates into hard or soft exudates which is done on the basis of threshold value.

vii) Mean, Median, Standard deviation are calculated and based on these features, the adaptive super pixel algorithm classifies the image of the eye as normal or diabetic eye.

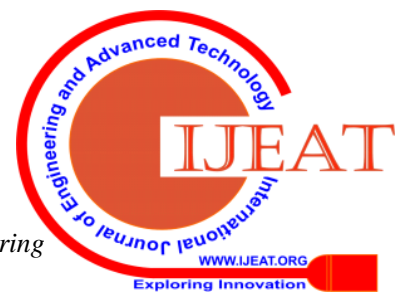




\section{RESULTS AND DISCUSSION}

\subsection{RESULTS AND DISCUSSION}

\section{Data Set Information:}

Dataset used is Messidor image for this research work. All features represent either a detected lesion, a descriptive feature of an anatomical part or an image-level descriptor.

The data set comprises 60 images including 25 diabetic retinopathy images and 35 healthy retinal images of size 256 x 256 pixels.

The input image 11 of a normal eye is taken from above data set and is shown in figure 6.1 is taken for processing.

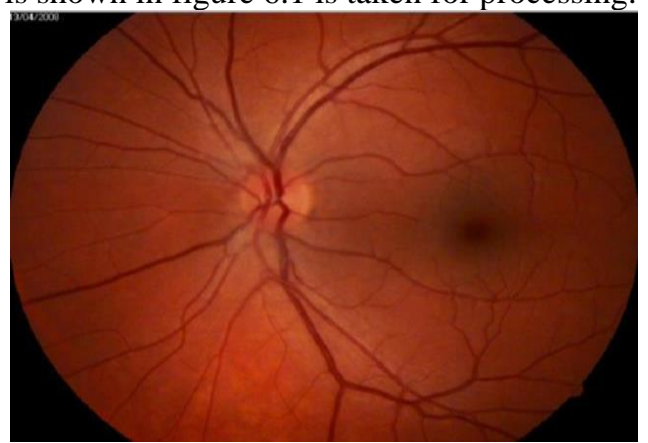

Figure 5.1 Normal eye

\subsubsection{Enhanced Image}

Image enhancement is accomplished by the use of filters, image editors and other tools to change the properties of retinal image of an eye.The enhanced image of normal eye is shown in figure 5.2 .

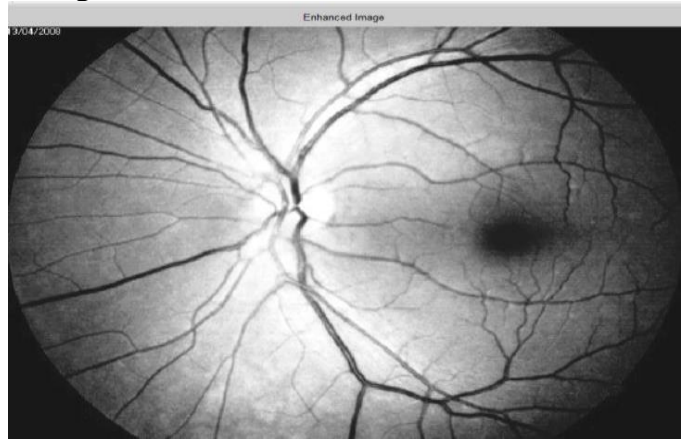

Figure 5.2 Enhanced image normal eye

\subsubsection{RGB Segmented Image}

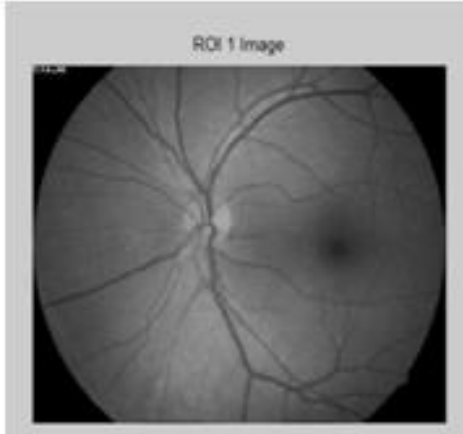

RGB segmentation is a process in which an image is divided into different regions in order to isolate the areas of interest on it.The RGB segmentation of normal eye is shown in figure 5.3 .

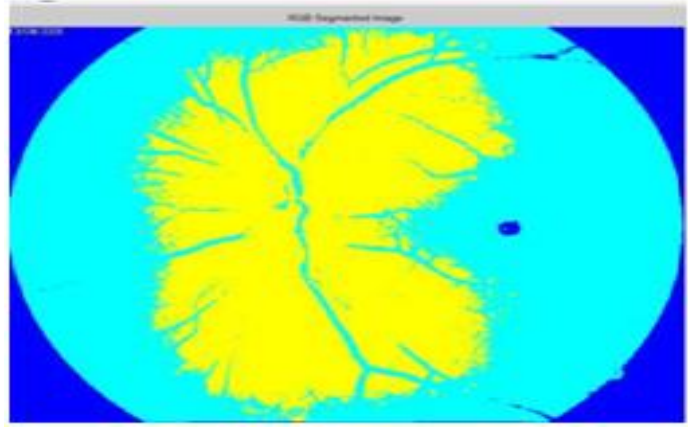

Figure 5.3 RGB segmentation of normal eye

\subsubsection{Optic Disk Location}

The optic disk location of normal eye is shown in figure 5.4

\section{Optic Disk}

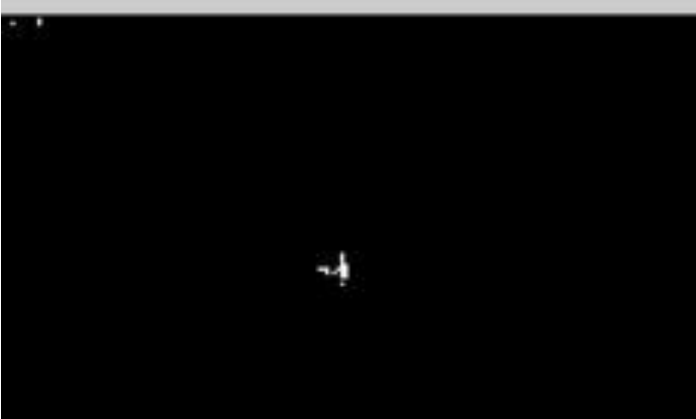

Figure 5.4 Optic disk location of normal eye The white point represents the location of optic disk in the image.

\subsubsection{Region of Interest}

In region of interest the boundaries of defected lesions of eye may be defined on an image or in a volume, for the purpose of measuring its size. The region of interest for red, green and blue channel of normal eye is shown in figure 5.5

Figure 5.5 Region of interest of normal eye

The green channel is used for its good contrast when compared with red and blue channel.

\subsubsection{Optic Disk Removal}

The green channel is taken for further processing from region of interest .The optic disk removed image of normal eye is shown in figure 5.6

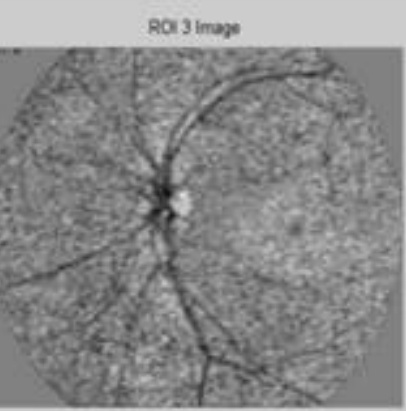




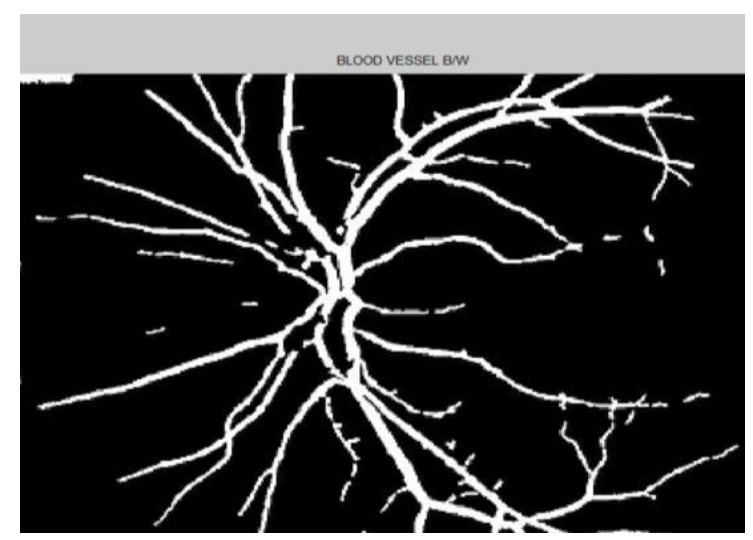

Figure 5.6 Optic disk removed image of normal eye

The optic disk is removed for better enhancement and classification

\subsubsection{Blood Vessel Extraction}

Blood vessel extraction is a processing step to extract vessels away from image to investigate the existence on some disease. The extracted blood vessel of normal eye is shown in figure 5.7 .

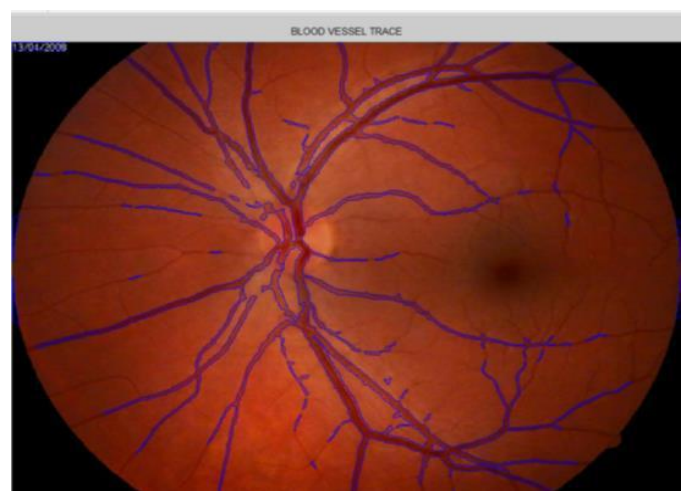

Figure 5.7 Blood vessel extracted image of normal eye
After optic disc removal the blood vessel trace is identified and removed for further classification

\subsubsection{Dots Part}

The white points and black points of normal eye is shown in figure 5.8. The occurrence of microaneurysm in eye is displayed as dots part.

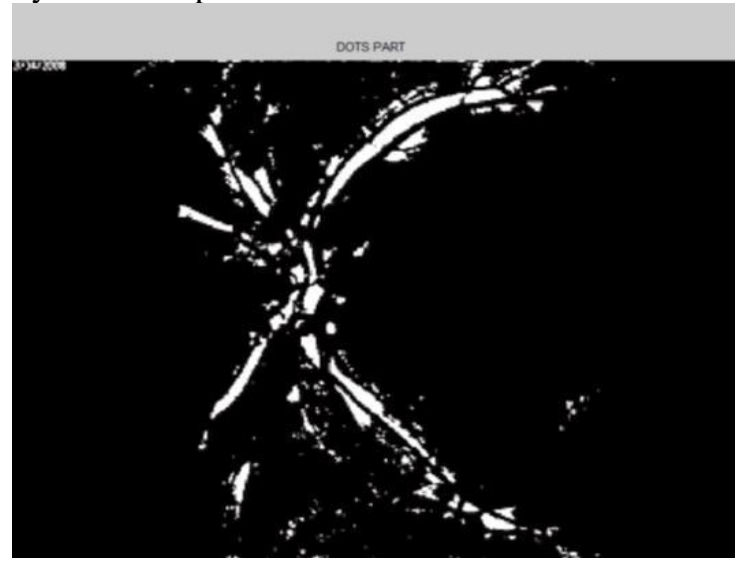

Figure 5.8 Dots part of normal eye

Based on the number of white points the eye is classified as normal and affected.

\subsubsection{Parameters measured}

The parameters like mean, standard deviation, entropy and maximum pixel points of normal eye are shown in table 5.1.

Table 5.1 Parameters of normal eye (Image 1)

\begin{tabular}{|c|c|c|c|c|c|c|}
\hline $\begin{array}{c}\text { Image } \\
\text { number }\end{array}$ & Deviation & $\begin{array}{c}\text { Maximum } \\
\text { pixel point }\end{array}$ & Entropy & $\begin{array}{c}\text { Standard } \\
\text { deviation }\end{array}$ & $\begin{array}{c}\text { Affected } \\
\text { pixel point }\end{array}$ & $\begin{array}{c}\text { Normal pixel } \\
\text { point }\end{array}$ \\
\hline 11 & 176.358 & 197.858 & 1.405 & 60.738 & 2.790 & 91422.30 \\
\hline
\end{tabular}

\subsection{RESULTS OF AFFECTED EYE}

\subsubsection{Input Image}

An affected eye is shown in figure 5.9 is taken for processing.

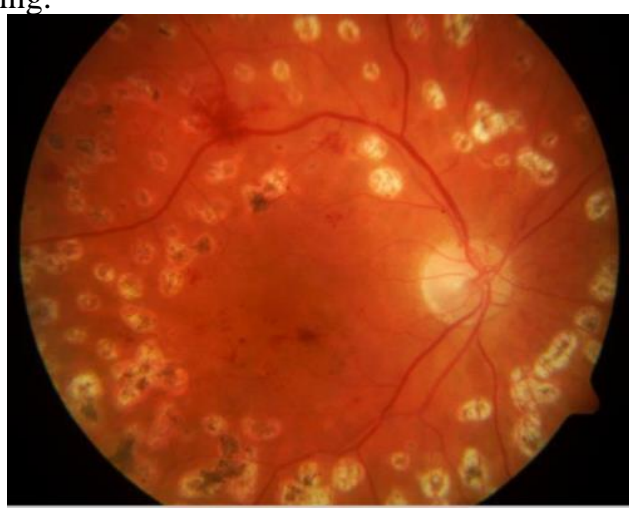

Figure 5.9 Image of affected eye

\subsubsection{Enhanced Image}

The enhanced image of affected eye is shown in figure 5.10

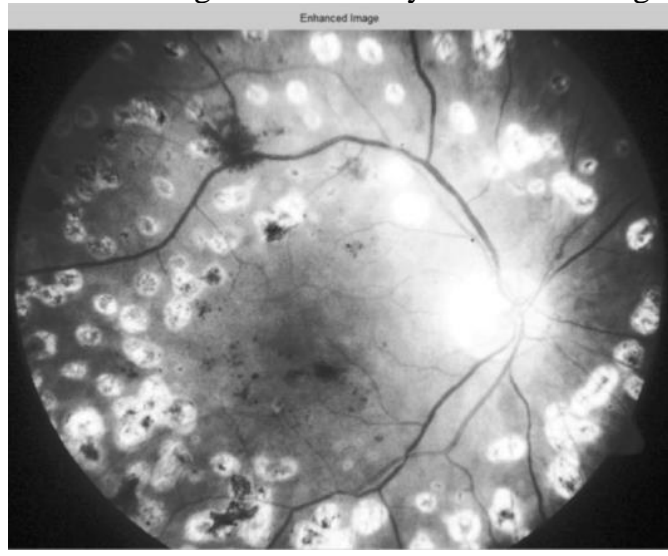

Figure 5.10 Enhanced image of affected eye

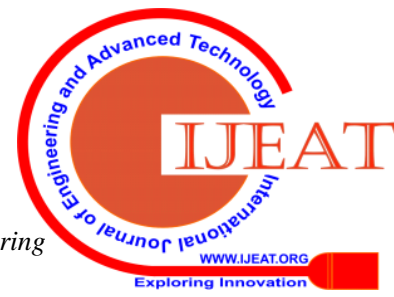




\subsubsection{RGB Segmentation}

The RGB segmentation of affected eye is shown in figure 5.11

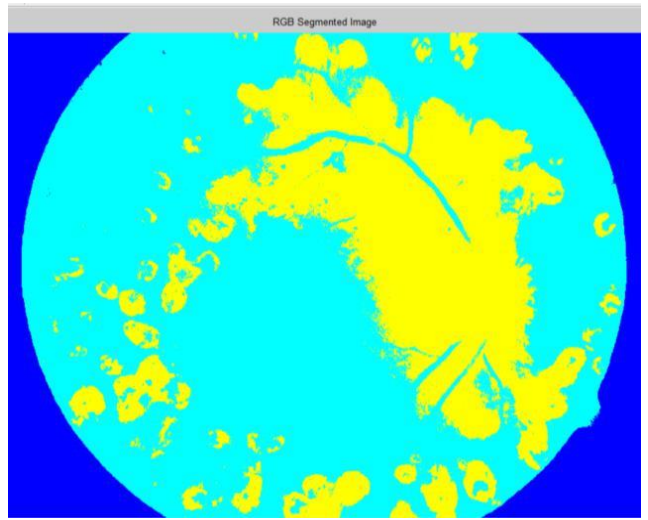

Figure 5.11 RGB segmentation of affected eye

The enhanced image is differentiated by RGB segmentation

\subsubsection{Optic Disk Location}

The optic disk location of affected eye is shown in figure 5.12 .

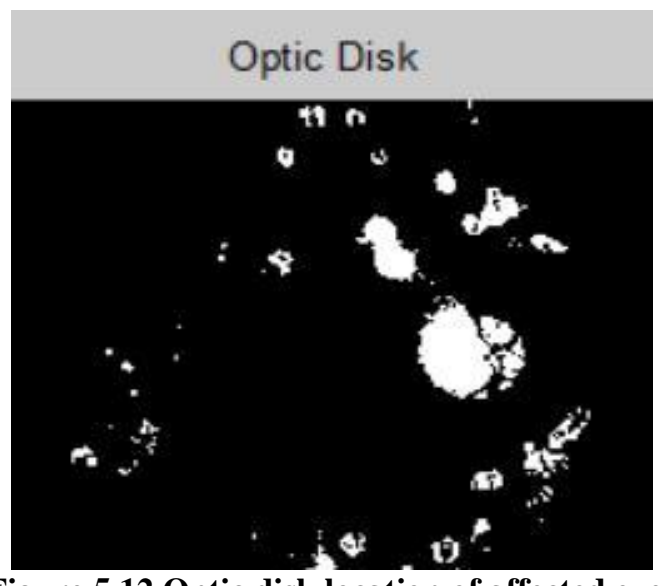

Figure 5.12 Optic disk location of affected eye

The white point represents the location of optic disk in the image.

\subsubsection{Region Of Interest}

The region of interest for red, green and blue channel of affected eye is shown in figure 5.13.

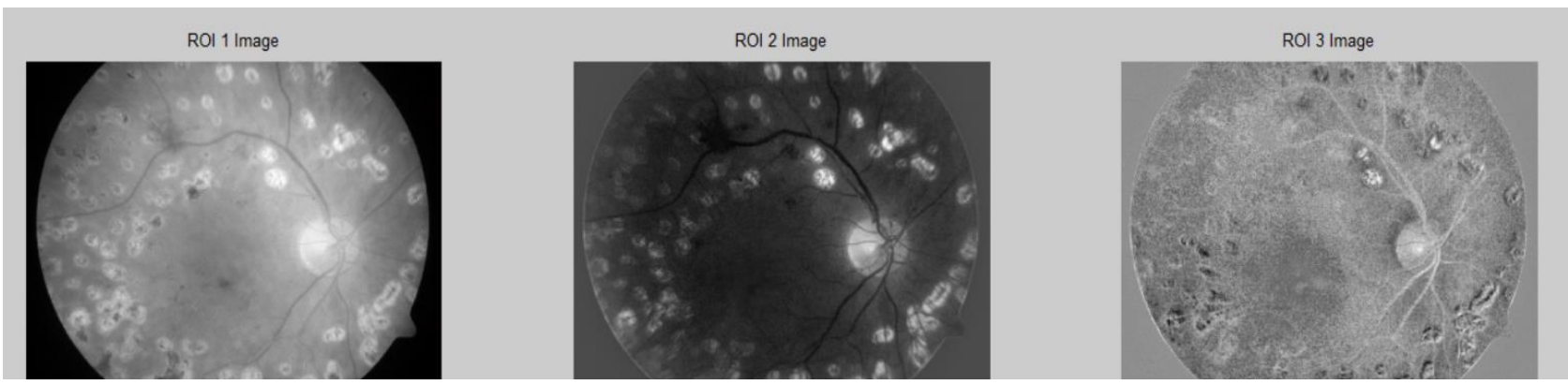

Figure 5.13 Region of interest of affected eye

The green channel is used for its good contrast when compared with red and blue channel.

\subsubsection{Optic Disk Removal}

The Optic disk removed image of affected eye is shown in figure 5.14.

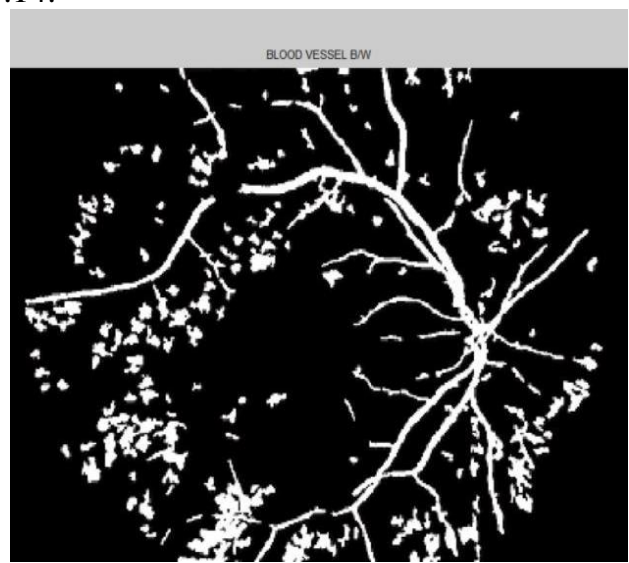

Figure 5.14 Optic disk removed image of affected eye

The green channel is taken for further processing from region of interest. The optic disk is removed for better enhancement and classification

\subsubsection{Blood Vessel Extraction}

The extracted blood vessel of affected eye is shown in figure 5.15 .

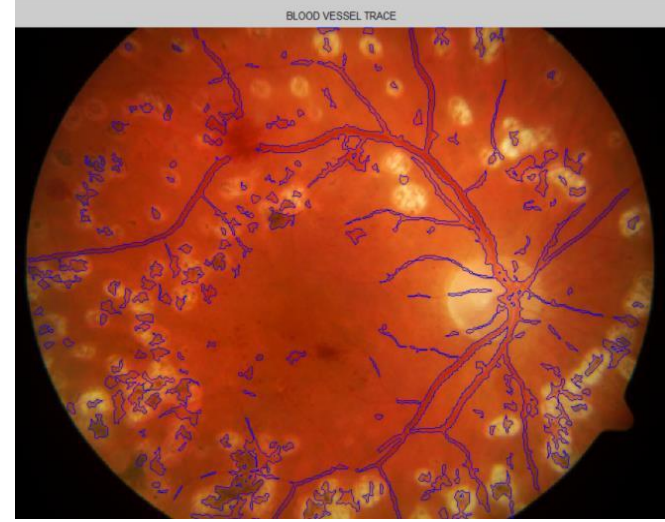

Figure 5.15 Blood vessel extracted image of affected eye

After optic disc removal the blood vessel trace is identified and removed for further classification

\subsubsection{Dots Part}

The white points and black points of affected eye is shown in figure 5.16

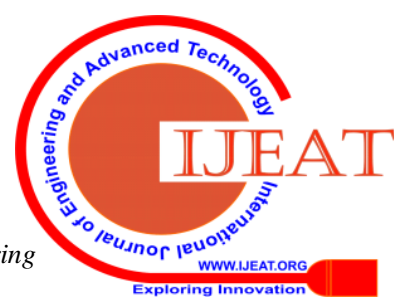




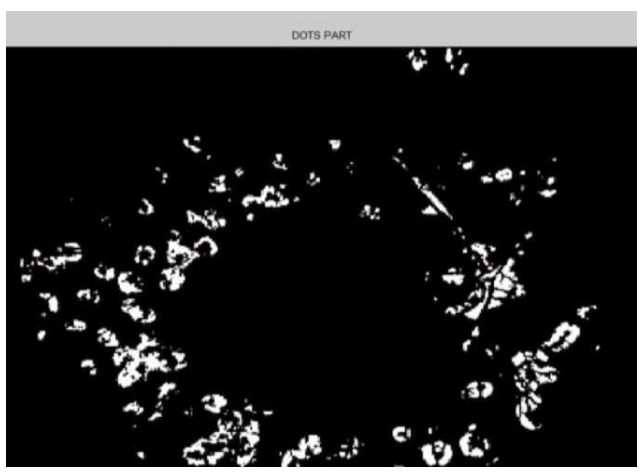

Figure 5.16 Dots part of affected eye

Based on the number of white points the eye is classified as normal and affected.

\subsubsection{Parameters measured}

The parameters of affected eye are shown in table 5.2 .

Table 5.2 Parameters of affected eye (Image 045)

\begin{tabular}{|c|c|c|c|c|c|c|}
\hline $\begin{array}{c}\text { Image } \\
\text { number }\end{array}$ & Deviation & $\begin{array}{c}\text { Maximum } \\
\text { pixel point Entropy }\end{array}$ & $\begin{array}{c}\text { Affected } \\
\text { Standard } \\
\text { pixel } \\
\text { point }\end{array}$ & $\begin{array}{c}\text { Normal } \\
\text { pixel } \\
\text { point }\end{array}$ \\
\hline $\begin{array}{c}\text { Image } \\
045\end{array}$ & 165.289 & 191.877 & 1.414 & 65.705 & 2590.582 & 88834.516 \\
\hline
\end{tabular}

\subsection{FINAL CLASSIFICATION AND RESULT}

By analyzing all the features and parameters the results are displayed as "INPUT IMAGE NORMAL (or) "INPUT IMAGE AFFECTED". The final result of normal is shown in figure 5.17 .
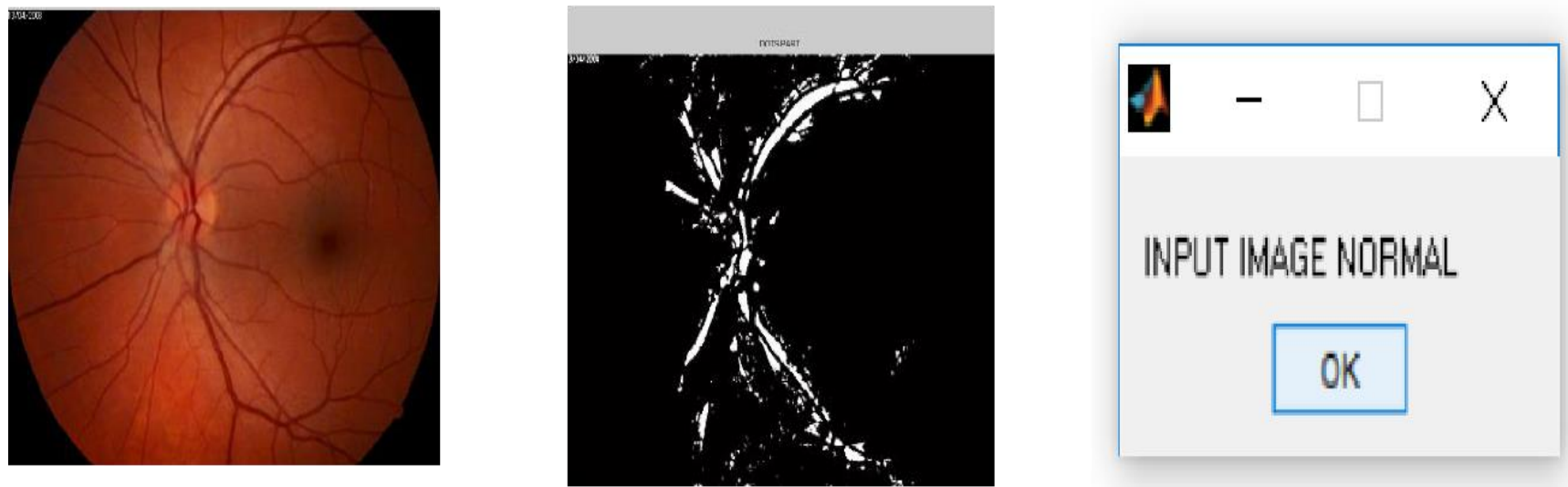

Figure 5.17 Final result of normal eye

The final result of affected eye is shown in figure 5.18
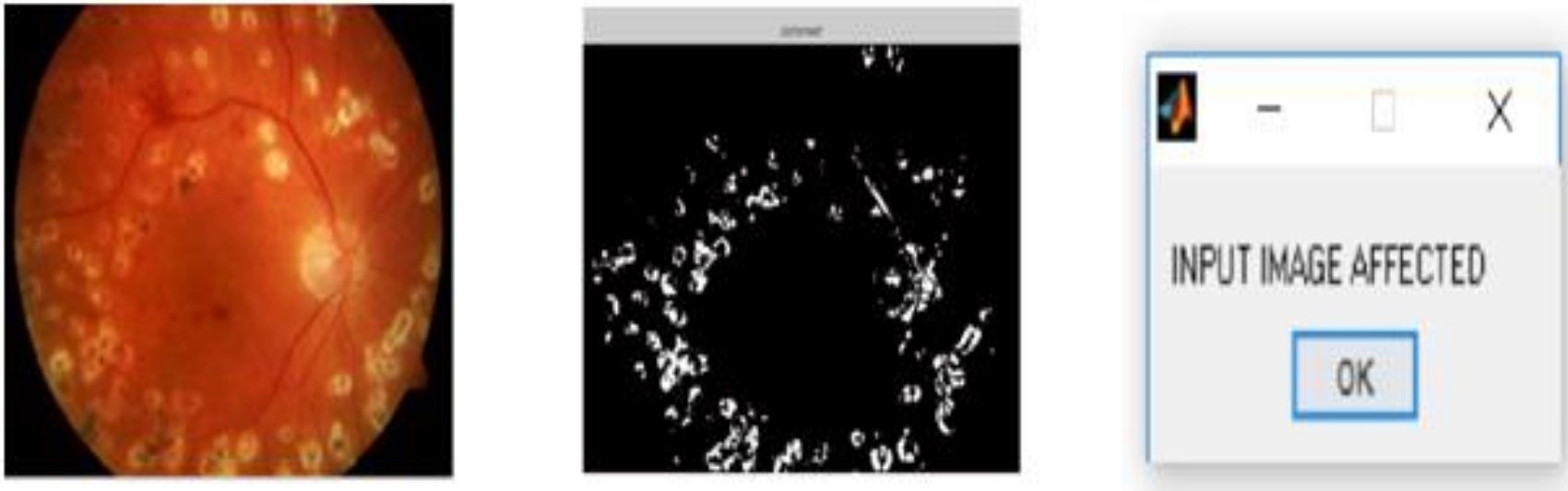

Figure 5.18 Final result of affected eye

In normal eye blood vessel there is no growth of blood vessel and no blood leakage.

Where as in abnormal eye there is more amount of blood leakage and emergence of new blood vessels.

\subsection{PARAMETERS}

5.4.1 Normal eye

Various parameters of all normal eye image in data set is shown in table 5.3.

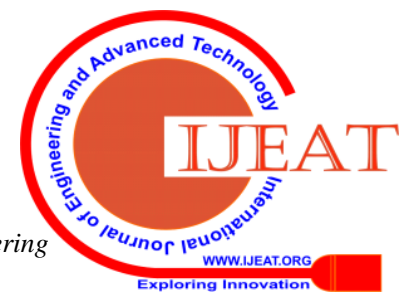


Table 5.3 Parameters of all normal eye image in data set

\begin{tabular}{|c|c|c|c|c|c|c|c|}
\hline$h$ & 8 & $\tau$ & $D$ & $t$ & 7 & $G$ & H \\
\hline s.o & IMAGE NUMBER & DEVIATIOX & MAXIMLI PIXEL POINT & ENTROPHY & STANDARD DEVIATION & AFFECTED PIXEL PONT & NORMAL PLEL POINTS \\
\hline 1 & 1 & 176.358 & 197.858 & 1.405 & 60.738 & 2791 & 91422.311 \\
\hline 2 & 4 & 182.986 & 214.569 & 1.412 & 62.647 & 4.185 & 91420.913 \\
\hline 3 & 7 & 182.418 & 215.719 & 1.416 & 62.555 & 4.185 & 91420.912 \\
\hline 4 & 8 & 180.46 & 211.802 & 1.422 & $61.80 ?$ & 16.74 & 91408.3 \\
\hline 5 & 10 & 182.712 & 211.386 & 1.414 & 62.575 & 279 & $91422.30^{\circ}$ \\
\hline 6 & 11 & 176.358 & 197.858 & 1.405 & 60.738 & 279 & 91422.312 \\
\hline 7 & 12 & 182.418 & 215.719 & 1.416 & 62.551 & 4.185 & 91420.912 \\
\hline 8 & 14 & 186.158 & 216.341 & 1.372 & 62.559 & 4.1851 & 91420.912 \\
\hline 9 & 15 & 181.13 & 210.979 & 1.421 & 62.082 & 279 & 91422.307 \\
\hline 10 & 16 & 179.5 & 2022218 & 1.421 & 61.484 & 122763 & 91302334 \\
\hline 11 & 18 & 174.931 & 201.1 & 1.378 & $\$ 9.711$ & 83702 & 91416,727 \\
\hline 12 & 21 & 182.969 & 217.491 & 1.413 & 62.976 & 57.1964 & 91367.901 \\
\hline 13 & 23 & 170.783 & 199.329 & 1.276 & 51.44 & 21602 & 91206.077 \\
\hline 14 & 24 & 183.834 & 214.212 & 1.405 & 62.929 & 219 & 91397.197 \\
\hline 15 & Inage 003 & 143.93 & 172238 & 1567 & 62.201 & 219.02 & 91206.077 \\
\hline 16 & leage 004 & 171.988 & 200.38 & 1.494 & 62391 & 0 & 91425.098 \\
\hline 17 & lenage 043 & 172.352 & 204.456 & 1.494 & 61.398 & 0 & 91425.098 \\
\hline 18 & Inaze 045 & 174.03 & 206.785 & 1.488 & 60.351 & 58.591 & 91366.506 \\
\hline 19 & leage 04? & 170.531 & 198.425 & 1.414 & 52.093 & 115.788 & 91309.31 \\
\hline \multirow[t]{2}{*}{20} & lmage 040 & 173.903 & 201.504 & 1.489 & 60.644 & 29.295 & 91395.802 \\
\hline & AVERAGE & 185.105 & 205.518 & 1.426 & 63,004 & 44.679 & 91379.96 \\
\hline
\end{tabular}

Ranges of parameters of normal eye :

Standard deviation lies in the range between 55 - 63 .

Affected pixel point lies in the range between $0-290$

Normal pixel point lies in the range between

91000 - 92000 as per reference paper[9].

\subsubsection{AFFECTED EYE}

Various parameters of all affected eye images in data set is shown in table 5.4 .

Table 5.4 Parameters of all affected eye images in data set

\begin{tabular}{|c|c|c|c|c|c|c|c|}
\hline S.No & DMAGE NUMBER & DEVLATION & MAXIMIM PLXEL POINT & ENTROPHY & STANDARD DEVLATION & AFFECTED PLEL POINT & NORMLAL PLEL POIN \\
\hline 1 & 3 & 160.879 & 182.347 & 0.694 & 70.851 & 412.93 & 91012. \\
\hline 2 & 9 & 172.341 & 204.94 & 1.34 & 69.106 & 756.109 & 90668. \\
\hline 3 & 12 & 182.48 & 211.305 & 1416 & 63.493 & 341.783 & 91083. \\
\hline 4 & 13 & 172.95 & 189.82 & 1.345 & 68.995 & 1037.906 & 90387. \\
\hline 5 & 17 & 180.533 & 203.272 & 1.422 & 63.872 & 313.883 & 9111: \\
\hline 6 & 19 & 184.382 & 211.209 & 1.399 & 63.066 & 729.633 & 90595. \\
\hline 7 & 20 & 180.604 & 206.455 & 1.424 & 63.181 & 1343.419 & 90754. \\
\hline 8 & 22 & 187.31 & 216.99 & 1349 & 63.913 & 671.012 & 90754. \\
\hline 9 & Image 035 & 170.804 & 200724 & 1.489 & 68.354 & 661.247 & 9076: \\
\hline 10 & Image 013 & 171.333 & 199.138 & 1492 & 68.881 & 3648.019 & $8 m 7$. \\
\hline 11 & Image 015 & 163.14 & 187.8 & 1.348 & 64.192 & 669.617 & 9075: \\
\hline 12 & Image 005 & 170.11 & 199.571 & 1.484 & 68.141 & 754.714 & 90670. \\
\hline 13 & Image 006 & 171.827 & 203.318 & 1.491 & 68.796 & 749.134 & 90675. \\
\hline 14 & Image 007 & 165.323 & 180.554 & 1.443 & 67.031 & 694.728 & 90730. \\
\hline is & Image 016 & 172.516 & 202.36 & 1.495 & 69.756 & 725.418 & 90699. \\
\hline 16 & Image 042 & 168.609 & 193.068 & 1.468 & 67.245 & 5868.917 & 8555: \\
\hline 17 & Image 044 & 162.506 & 190.647 & 1.415 & 66.99 & 636.136 & 90788. \\
\hline 18 & Image 019 & 173.838 & 202.31 & 1.489 & 70.17 & 876.082 & 90549 \\
\hline \multirow[t]{2}{*}{19} & Image 017 & 169.598 & 200.356 & 1.482 & 68.048 & 362.239 & 91042 \\
\hline & AVERAGE & 163.705 & 189.309 & 1.324 & 63.689 & 1054.69 & 85565 \\
\hline
\end{tabular}

Ranges of parameters of affected eye :

Standard deviation lies in the range between 63-75. Affected pixel point lies in the range of 290 and above

Normal pixel point lies in the range of 91000 and below as per reference paper[9].

\subsection{DOCTOR'S OPINION FOR NORMAL EYE}

Doctor's confirmation for normal eye is shown in table 5.5

Table 5.5 Doctor's verification for normal eye

\begin{tabular}{|c|c|c|c|c|}
\hline $\begin{array}{c}\text { IMAGE } \\
\text { NUMBER }\end{array}$ & $\begin{array}{c}\text { STANDARD } \\
\text { DEVIATION }\end{array}$ & $\begin{array}{c}\text { AFFECTED } \\
\text { PIXEL } \\
\text { POINT }\end{array}$ & $\begin{array}{c}\text { NORMAL } \\
\text { PIXEL } \\
\text { POINTS }\end{array}$ & $\begin{array}{c}\text { DOCTOR' } \\
\text { S } \\
\text { OPINION }\end{array}$ \\
\hline 1 & 60.738 & 2.791 & 91422.311 & YES \\
\hline 4 & 62.647 & 4.185 & 91420.913 & YES \\
\hline
\end{tabular}

\begin{tabular}{|c|c|c|c|c|}
\hline 7 & 62.555 & 4.185 & 91420.912 & YES \\
\hline 10 & 62.575 & 2.79 & 91422.307 & YES \\
\hline Image 004 & 62.391 & 0 & 91425.098 & YES \\
\hline Image 047 & 52.093 & 115.788 & 91309.31 & YES \\
\hline Image 040 & 60.644 & 29.295 & 91395.802 & YES \\
\hline 8 & 61.807 & 16.74 & 91408.3 & YES \\
\hline 11 & 60.738 & 2.79 & 91422.321 & YES \\
\hline 12 & 62.551 & 4.185 & 91420.912 & YES \\
\hline $\mathbf{1 4}$ & $\mathbf{6 2 . 5 5 9}$ & $\mathbf{7 5 4 . 7 1 4}$ & $\mathbf{9 0 6 7 0 . 3 8 3}$ & NO \\
\hline 15 & 62.082 & 2.79 & 91422.307 & YES \\
\hline
\end{tabular}




\section{REFERENCES}

\begin{tabular}{|c|c|c|c|c|}
\hline 16 & 61.484 & 122.763 & 91302.334 & YES \\
\hline 18 & 59.7111 & 8.3702 & 91416.727 & YES \\
\hline 21 & 62.976 & 57.1964 & 91367.901 & YES \\
\hline 23 & 51.44 & 216.02 & 91206.077 & YES \\
\hline 24 & 62.929 & 21.9 & 91397.197 & YES \\
\hline Image 003 & 62.201 & 219.02 & 91206.077 & YES \\
\hline Image 043 & 61.398 & 0 & 91425.098 & YES \\
\hline
\end{tabular}

\subsection{DOCTOR'S OPINION FOR AFFECTED EYE}

Doctor's confirmation for affected eye is shown in table 5.6

Table 5.6 Doctor's verification for affected eye

\begin{tabular}{|c|c|c|c|c|}
\hline $\begin{array}{c}\text { IMAGE } \\
\text { NUMBER }\end{array}$ & $\begin{array}{c}\text { STANDARD } \\
\text { DEVIATION }\end{array}$ & $\begin{array}{c}\text { AFFECTED } \\
\text { PIXEL } \\
\text { POINT }\end{array}$ & $\begin{array}{c}\text { NORMAL } \\
\text { PIXEL } \\
\text { POINTS }\end{array}$ & $\begin{array}{c}\text { DOCTOR'S } \\
\text { CONFIRMA } \\
\text { TION YES }\end{array}$ \\
\hline 9 & 69.106 & 756.109 & 90668.988 & YES \\
\hline 13 & 68.995 & 1037.906 & 90387.191 & YES \\
\hline & 68.881 & 3648.019 & 87777.078 & YES \\
\hline Image 013 & & & & \\
\hline & 67.246 & 5868.917 & 85556.18 & YES \\
\hline Image 042 & & & & \\
\hline & 68.141 & 754.714 & 90670.383 & YES \\
\hline Image 005 & & & & \\
\hline 20 & 63.181 & 1343.419 & 90754.085 & YES \\
\hline & 66.99 & 636.136 & 90788.961 & YES \\
\hline Image 044 & & & & \\
\hline 3 & 70.851 & 412.93 & 91012.167 & YES \\
\hline 12 & 63.493 & 341.783 & 91083.314 & YES \\
\hline 17 & 63.872 & 313.883 & 91111.21 & YES \\
\hline 20 & 63.181 & 1343.419 & 90754.085 & YES \\
\hline 22 & 63.913 & 671.012 & 90754.085 & YES \\
\hline Image 035 & 68.354 & 661.247 & 90763.85 & YES \\
\hline Image 015 & 64.192 & 669.617 & 90755.48 & YES \\
\hline Image 006 & 68.796 & 749.134 & 90675.963 & YES \\
\hline Image 007 & 67.031 & 694.728 & 90730.369 & YES \\
\hline Image 016 & 69.756 & 725.418 & 90699.678 & YES \\
\hline Image 042 & 67.246 & 5868.917 & 85556.8 & YES \\
\hline 59 & & & \\
\hline
\end{tabular}

59 images out of 60 images gives the exact result. Thus, the accuracy of the project is $98.33 \%$

\section{CONCLUSION AND FUTURE WORK}

Screening of diabetic retinopathy automatically is very difficult and it is important as it helps the eye specialists to diagnose the various grades of diabetic retinopathy during the examination of the eye which is accomplished by the combination of various steps such as the integrated use of morphological operations along with adaptive super pixel algorithm. This helps doctors to cure patients at the earlier stage and also prevents eye transplantation. The severity of the diabetic retinopathy can be further graded based on the detected lesions and their quantities. The performances of the method were also measured by specificity $95 \%$ and accuracy 98.33\%.In the future work, identifying more features that will improve the performance of adaptive super pixel algorithm. The accuracy can also be increased by improving the performance of the data or even by algorithm tuning. In addition, this system can be used to detect other retinal diseases.
1. https://www.allaboutvision.com/en-in/conditions/diabetic/

2. https://www.ncbi.nlm.nih.gov/pmc/articles/PMC2613584/

3. https://www.niddk.nih.gov/health-information/diabetes/overview/wha t-is-diabetes

4. https://www.iapb.org/wp-content/uploads/Guidelines-for-the-Compre hensive-Management-of-DR-in-India.pdf

5. Salz, D. A., \& Witkin, A. J. (2015). Imaging in diabetic retinopathy. Middle East African journal of ophthalmology, 22(2), 145-150. doi:10.4103/0974-9233.151887

6. Sreng, Syna, Noppadol Maneerat, Kazuhiko Hamamoto and Ronakorn Panjaphongse. "Automated Diabetic Retinopathy Screening System Using Hybrid Simulated Annealing and Ensemble Bagging Classifier.' (2018). DOI:10.3390/app8071198

7. Manoj Kakarla (2016), "Optimal Feature Extraction and Classification of Diabetes using Naïve Bayesian Classifier", International Journal of Engineering Trends and Technology (IJETT), Vol-42, pp 63-70.

8. K. R. Ananthapadmanaban and G.Parthiban (2014), "Prediction of Chances - Diabetic Retinopathy using Data Mining Classification Technique", Indian Journal of Science and Technology, Vol 7, pp 1498-1503.

9. S, Kandiraju N, Thompson HW. Design and implementation of a unique blood-vessel detection algorithm towards early diagnosis of diabetic retinopathy. In: International conference on information technology: coding and computing, vol. 1. 2005. p. 26-31.

10. http://www.kscst.iisc.ernet.in/spp/39_series/SPP39S/02_Exhibition_P rojects/177_39S_BE_1679.pdf

11. A. B. Chavan (2017), " Image Processing Analysis on Retina Blood Vessel for Detecting Glaucoma", International Journal of Electronics, Communication \& Soft Computing Science and Engineering, Vol. 45, Issue 1, pp 63-85.

12. Abramoff, M.D and Garvin, M.K. (2010), "Retinal Imaging and Image Analysis", IEEE Reviews in Biomedical Engineering, Vol. 3, pp 12-20.

13. Aiswarya Iyer (2015), "Diagnosis of diabetes using classification mining techniques", International Journal of Data Mining \& Knowledge Management Process Vol.5, pp 52-57.

14. C. E. Marwan D. Saleh (2012), "An automated decision support system for non-proliferative diabetic retinopathy disease based on MAs and HAs detection", Computer Methods and Programs in Biomedicine, Vol. 108, pp. 186-196.

15. G. D. Joshi, J. Sivaswamy, and S. R. Krishnadas (2011), “Optic Disk and Cup Segmentation from Monocular Colour Retinal Images for Glaucoma Assessment,” IEEE Trans. Medical Imaging, Vol. 30, no. 6, pp. 1192-1205.

16. Huiling Chen (2017), "Automatic Analysis of Microaneurysms Turnover to Diagnose the Progression of Diabetic Retinopathy", Institute of Electrical and Electronics Engineers, Vol - 8, pp 976-997.

17. Kevin Noronha (2015), "Support System for the Automated Detection of Hypertensive Retinopathy using Fundus Images", International Conference on Electronic Design and Signal Processing, Vol. 19, pp 567-589.

18. Mohammed Ghazal (2016), "Early Diagnosis of Diabetic Retinopathy in OCTA Images Based on Local Analysis of Retinal Blood Vessels and Foveal Avascular Zone", International Conference on Pattern Recognition (ICPR), Vol.5, pp 623-647.

19. Muhammad Nauman Zahoora (2018), "Fast Optic Disc Segmentation in Retina Using Polar Transform”, International Journal of Engineering Trends and Technology (IJETT), Vol - 6, pp 12-18.

20. Syna Sreng and Noppadol Maneerat (2018), "Primary Screening of Diabetic Retinopathy Based on Integrating Morphological Operation and Support Vector Machine”, International Conference on Intelligent Informatics and Biomedical sciences, Vol - 3, pp 1- 3 .

21. T. Teng, et al. (2002), "Progress towards automated diabetic ocular screening: a review of image analysis and intelligent systems for diabetic retinopathy", Medical and Biological Engineering and Computing, Vol. 40, pp. 2-13.

22. Tamilselvan, KumaravelSubramaniam, and GovindasamyMurugesan. "Survey and analysis of various image fusion techniques for clinical CT and MRI images." International Journal of Imaging Systems and Technology vol.24, no.2, pp.193-202, 2014

23. Xiaolin Xiao, Yue-Jiao Gong, Yicong Zhou. "Adaptive superpixel segmentation aggregating local contour and texture features", 2017 IEEE International Conference on Acoustics, Speech and Signal Processing (ICASSP), 2017

Published By:

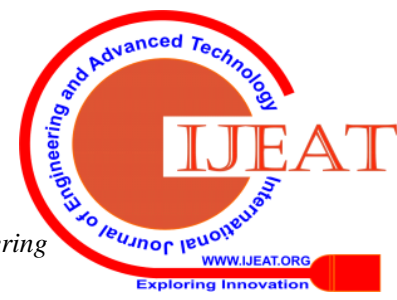




\section{AUTHORS PROFILE}

Dr. S. Balambigai received her B.E. degree in ECE from Bharathiar University with distinction, M.E. (Applied Electronics) and Ph.D from Anna University, Chennai. She has around 35 publications in reputed national and international and National journals/conferences and three patent to her credit. She has a total of 16 years experience and is currently working as an Associate Professor in the Department of Electronics and Communication Engineering in Kongu Engineering College. Developments in biomedical engineering and computer networks keeps her fascinated to carry on further research in the these domains.

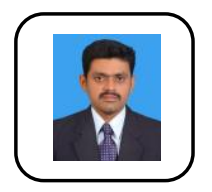

Dr. V. Saravanan is currently working as an Associate Professor in the Department of Computer Science and Engineering, K L Deemed to be University,Koneru Lakshmaiah Education Foundation, Guntur,Andhra Pradesh, India. He completed M.E. and Ph.D.degrees in Computer Science and Engineering from AnnaUniversity, Chennai, Tamilnadu, India. He has published 20+ Journal papers and 5+ patents. His research interest includes Wireless Networks, Mobile Computing, and Security.

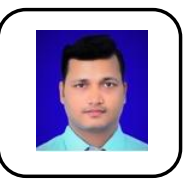

Dr. Rudra Kalyan Nayak is presently working as Associate Professor in the Department of Computer Science \& Engineering at KoneruLakshmaiah Education Foundation (Deemed to be University), Green Fields, Vaddeswaram, Andhra Pradesh, India. He has got his M.Tech in Information Technology and Ph.D in Computer Science \& Engineering from Siksha ' $O$ ' Anusandhan (Deemed to be) University, Odisha, India. He has together more than 10 years of teaching, mentoring and research experience. His research interest lies in the field of Artificial Intelligence, Financial Engineering, Bioinformatics and Computer Vision. He is professional member of ISTE and ACM.

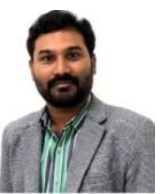

Dr. T. Gunasekaran received his B.E. degree in ECE from Bharathiar University , M.E. (Communication Engineering) from Birla Institute of Technology and Science, Pilani and Ph.D (Information and Communication Engineering) from Anna University, Chennai. He has around 22 publications in reputed National and International journals/conferences and one patent to his credit. He has a total of 19 years experience in teaching \& research and is currently working as Program Director in the Department of Engineering- EEE Section, Electronics and Communication Engineering in Higher College of Technology, Muscat, Sultanate of Oman. His area of interest includes Antenna design

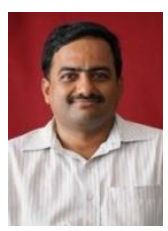

S. Hariprasath has received B.E degree in 2000 and Masters in Communication Systems in 2008. He has published papers in 8 IEEE conferences, 7 International conferences. He has published 1 paper in national journal, 4 papers in international journals (annexure 2), and 1 international journal publication. He has published 1 paper in Science Citation Indexed Journal (SCI) to his credit. He is currently pursuing research in Pattern Recognition in VLSI. He has 16 years of teaching experience. He has guided more than 14 PG projects and 30 UG projects in the areas of Image processing, VLSI and Robotics. He has received Special Prize for contribution in eYRC15. His areas of interest include Biometrics, Pattern Recognition, FPGA Programming. He works as Assistant Professor in Department of Electronics and Communication Engineering , Saranathan College of Engineering, Trichy. 